\title{
La prise en charge de la santé par les associations du quartier Biyem Assi (Yaoundé)
}

Séverin Cécile Abéga et Noël Solange Ngo Yebga

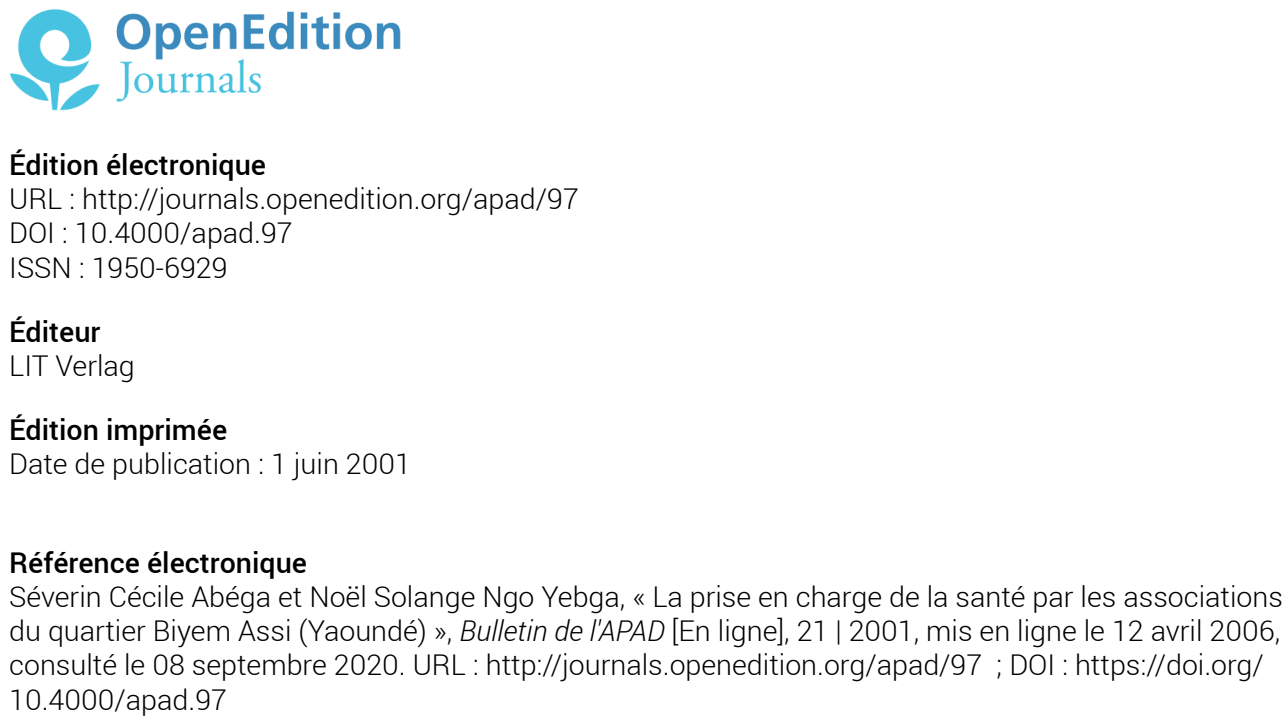

Édition électronique

URL : http://journals.openedition.org/apad/97

DOI : $10.4000 /$ apad. 97

ISSN : 1950-6929

Éditeur

LIT Verlag

Édition imprimée

Date de publication : 1 juin 2001

Référence électronique

Séverin Cécile Abéga et Noël Solange Ngo Yebga, «La prise en charge de la santé par les associations du quartier Biyem Assi (Yaoundé) », Bulletin de l'APAD [En ligne], 21 | 2001, mis en ligne le 12 avril 2006, consulté le 08 septembre 2020. URL : http://journals.openedition.org/apad/97 ; DOI : https://doi.org/ 10.4000/apad.97

Ce document a été généré automatiquement le 8 septembre 2020.

Bulletin de l'APAD 


\title{
La prise en charge de la santé par les associations du quartier Biyem Assi (Yaoundé)
}

\author{
Séverin Cécile Abéga et Noël Solange Ngo Yebga
}

1 L'appartenance aux associations est une réalité importante dans le milieu urbain camerounais. La plupart des individus sont membres d'une ou de plusieurs associations. S'inscrire dans une association peut donc se justifier par les difficultés qu'éprouvent les populations qui sont d'ordre économique, sanitaire, social, mais aussi par des préoccupations personnelles et communes liées aux diverses activités politiques, économiques, sociales et juridiques qu'offrent ces structures. D'autres aspects sont aussi apparus, notamment sur le plan de l'acculturation, du changement social, de la sociologie urbaine, auxquels se mêlent des questions relatives au genre.

2 L'adhésion à ces entités se fait parfois d'une manière volontaire ou de gré à gré par l'intermédiaire d'un ami, d'un voisin, d'un collègue de service, d'un ancien camarade de collège, d'un "frère" du village ou d'une même région déjà installé en ville. Ce dernier initie alors le nouvel arrivant à la réalité urbaine, l'insérant dans un réseau de solidarité sélective dont l'appartenance exige en permanence des dons et l'acceptation de contre dons, ainsi que la participation régulière à des réunions, à des cérémonies et à des rituels collectifs. Il ne se crée pas seulement, à travers ces regroupements des stratégies de survie, dont l'importance se fait sentir en cas d'urgence de maladie et surtout de deuil, mais toute une dynamique par laquelle des modes de pensée venus des villages s'adaptent à la ville, des manières de faire venues d'ailleurs se transforment pour s'immerger dans les agir locaux, avant leur transfert vers les villages.

La problématique des associations nous permet alors de nous poser la question de savoir si elles peuvent être des acteurs dans un domaine précis, celui de la santé, qui met en évidence la précarité des populations et la vulnérabilité des individus. En nous intéressant particulièrement à la prise en charge des soins de santé, nous essayons de voir comment cette transformation sociale se met en œuvre dans un domaine délicat et sensible, et si l'action des groupes permet déjà, ou peut laisser espérer un meilleur 
accès aux soins. Nous essayons ainsi d'identifier les domaines dans lesquels s'oriente l'action de ces groupes, et la manière dont laquelle ils interviennent dans le champ de la santé.

Caractéristiques des associations

4 La multiplicité des associations rencontrées permet de mettre en évidence les facteurs essentiels de ces groupes qui se caractérisent par :

- un certain nombre de membres recrutés sur une base qui peut être ethnique, religieuse ou culturelle, nous y reviendrons

- des activités parmi lesquelles des réunions, des échanges de prestations financières, sociales, religieuses, de services organisées au cours des réunions

- un cycle précis qui organise les activités sur une période qui est généralement d'un an, ponctuée de réunions qui peuvent être mensuelles, bimensuelles, hebdomadaires certaines femmes, notamment les revendeuses de vivres frais, et même les prostituées, peuvent organiser des réunions quotidiennes

- l'implication dans la vie des membres par la participation aux fêtes, aux rituels, et l'assistance matérielle, entre autres dans le domaine de la santé

- la consommation collective de boissons, de nourritures, le port d'uniformes, la création de chants, de danses, de slogans, etc.

- les cérémonies de fin de cycle pour "casser" les "caisses", c'est-à-dire remettre tous les comptes à niveau, et lancer le calendrier des activités du cycle suivant

5 La création des associations en général n'obéit à aucun critère prédéfini. Le plus souvent, l'orientation tient compte des objectifs formulés et recherchés par les membres créateurs. Il en existe d'autres qui ne sont pas verbalisés, mais qui n'en existent pas moins, et imprègnent fortement toute l'action des associations. Progressivement, l'association s'adapte en fonction de l'environnement social et économique quand ces autres objectifs s'imposent, consciemment ou non, aux membres. L'enquête réalisée à Biyem Assi nous montre les associations créées sur des bases variées. Celles qui ont été expressément formulées sont les suivantes :

- la religion

- la camaraderie

- la famille

- la région

- l'amitié

- le milieu socio-professionnel

- le sport

- l'ethnie

6 L'entraide et la solidarité sont les bases fondamentales de ces différents groupements, et prennent la forme d'une assistance financière ou psychologique qui va au-delà des solidarités préétablies par la religion ou l'appartenance à un groupe ethnique.

7 Parmi les membres des associations interrogés, les Beti et les Bamiléké sont les plus nombreux. Cela peut venir en partie remettre en question l'idée reçue au Cameroun selon laquelle la mentalité associative caractériserait les Bamiléké, et serait inconnue des Beti. En fait, Beti et Bamiléké sont les ethnies les plus nombreuses et les mieux représentées dans les villes du Cameroun au sud de l'Adamaoua. Les Bassa, ethnie de moyenne importance au Cameroun, viennent immédiatement après. Relevons également l'existence d'une association de Massa, groupe de l'Extrême-Nord camerounais, dans la région dite du "bec de canard", à la frontière de la République du 
Tchad, et dont les originaires étaient peu nombreux dans les villes du sud du Cameroun. Les départements de la province de l'Ouest sont les plus représentés ; les originaires de cette province se rencontrent aussi bien dans les associations à caractère régional, ethnique, familial que dans les associations de quartier.

8 Si on a parlé d'une "mentalité associative des Bamiléké", et si les Bamiléké semblent être les plus nombreux dans les associations, c'est sans doute bien davantage en raison des densités de peuplement de leur région, l'importance numérique étant alors significative. Il faut aussi compter avec les stratégies économiques liées aux densités démographiques. La pression démographique est très forte dans la province de l'ouest, dont sont originaires les Bamiléké, et dans le département de la Lékié, d'où viennent les Eton, l'un des sous-groupes Beti les plus nombreux. Les terres manquent de ce fait, et les cadets sociaux sont littéralement expulsés en raison des stratégies successorales et des dynamiques foncières qui permettent aux aînés de s'approprier les terres. De ce fait, pour de nombreuses personnes, l'exode rural, surtout vers les villes, devient une solution, et si l'on continue de se réclamer d'un village, on investit plus en ville, où se construisent le présent et l'avenir. Pour cette couche de la population, la ville n'est plus seulement l'endroit où l'on travaille et où l'on gagne de l'argent; on commence à appartenir à l'endroit où l'on est, parce que l'inscription en fonction du lieu de naissance et de la terre des ancêtres devient problématique. Les stratégies d'insertion au milieu urbain prennent donc une importance plus singulière, et les associations, auxquelles ce rôle est dévolu prennent un relief particulier. Cette donnée particulière permet de mieux comprendre pourquoi les originaires des départements où vit l'ethnie bamiléké s'inscrivent autant dans les associations, et pourquoi on reconnaît tant de vitalité aux associations bamiléké, qui brillent par leur longévité, leur inventivité, leur discipline, et l'importance que leurs membres leur accordent. Il s'agit là de l'attitude de personnes qui ont une véritable stratégie de vie citadine, et pour lesquels la ville prend peu à peu une importance supérieure à celle du village.

9 Les membres des associations se regroupent pour plusieurs raisons. Les bases de regroupement peuvent être religieuses, familiales, régionales pour les originaires d'une même province, d'un même département, d'un même village, ou parce qu'ils veulent mener ou mènent des activités ensemble : les amicales, les sportifs, les collègues, les coreligionnaires. On découvre donc que les références ne sont pas seulement un passé commun (ethnie, région, famille). Elles peuvent être aussi un présent commun, un futur qu'on envisage ensemble (amicales, sportifs, collègues, voisinage). Il va cependant sans dire que ces bases se recoupent, et on peut être voisin, et décider de se réunir parce qu'on appartient à la même ethnie; ou appartenir à la même ethnie, et décider de s'associer pour mener des activités d'intérêt commun.

10 La référence ethnique est la plus fréquente, mais elle n'est ni unique, ni majoritaire. Les autres éléments de regroupements ne doivent donc pas être négligés, car il semble bien apparaître ici que l'inscription régionale laisse une large place à d'autres éléments référentiels. Les solidarités à Biyem Assi sont donc multiples, et cela est caractéristique d'une citadinité aux inscriptions sociales diversifiées. A Biyem Assi, tout le monde a une tribu, mais on convoque le voisinage, la religion, la profession, l'activité sportive au moment de construire les solidarités.

11 Notre enquête fait apparaître que les associations féminines sont les plus nombreuses, et qu'il y a trois fois plus d'évocations d'associations typiquement féminines que d'associations masculines, la mixité étant au même niveau que la dynamique féminine. 
Les femmes s'organisent en s'appuyant beaucoup sur l'ethnie, mais aussi sur d'autres bases, par exemple, en se référant aux études dans un même établissement, à l'assistance envers les femmes rurales de sa région ou de son village, etc. La vie associative est donc au cœur des stratégies des femmes de Biyem Assi. De plus, celles-ci s'y investissent dans une certaine mesure pour augmenter leur pouvoir d'achat et subvenir aux besoins du ménage. Les hommes préfèrent d'ailleurs les associations gérées par des femmes, et l'un des postes qu'on leur confie le plus souvent est celui de trésorier. Beaucoup d'hommes participent aux associations par l'intermédiaire de leur épouse, lui donnant l'argent pour les cotisations et l'épargne, et lui laissant le soin de le gérer. La plupart des femmes sont membres de plusieurs associations. Elles manifestent aussi leur inscription par le port d'un uniforme, généralement une ample robe de cotonnade très caractéristique, appelée localement kabangondo, et popularisée par les femmes Douala, d'où le nom ngondo, qui désigne un rituel collectif de l'ethnie Douala. En dehors de tous les objectifs avoués, les réunions des associations donnent aux femmes l'occasion de chanter, y compris des chansons très lestes, de danser, y compris des danses très suggestives, de brocarder les époux et les ennemies, de boire et donc de sortir du cadre très contraignant de la vie familiale et au sein du ménage, de s'écarter un peu des normes, de questionner les règles sociales, les contraintes. Ces réunions constituent donc un moment très important, aussi bien sur le plan social que psychologique, et mesurer leur importance uniquement en termes économiques est très réducteur.

Tableau 1 : Raisons déclarées d'adhésion à une association

\begin{tabular}{|l|l||l|l|l|l|l|l|l|}
\hline $\begin{array}{l}\text { Entraide } \\
\text { assistance }\end{array}$ & $\begin{array}{l}\text { Faire } \\
\text { connais- } \\
\text { sance }\end{array}$ & $\begin{array}{l}\text { Amitié, } \\
\text { camara- } \\
\text { derie }\end{array}$ & $\begin{array}{l}\text { Sport } \\
\text { Dévelop- } \\
\text { pement }\end{array}$ & $\begin{array}{l}\text { Education } \\
\text { Sensibi- } \\
\text { lisation }\end{array}$ & $\begin{array}{l}\text { Relations de } \\
\text { voisinage }\end{array}$ & $\begin{array}{l}\text { Évangéli- } \\
\text { sation }\end{array}$ & Total \\
\hline \hline 37 & 5 & 3 & 3 & 2 & 5 \\
\hline
\end{tabular}

12 Les buts des associations sont multiples et variés. Ils se résument souvent pour certaines à renforcer les liens de famille et des relations de bon voisinage, à participer au développement et assurer la promotion des terroirs d'origine, à éduquer leurs membres, à s'associer pour la construction d'écoles dans la région d'origine ou la construction de locaux pouvant accueillir les manifestations à caractère culturel tel qu'un congrès annuel d'originaires, ou mener ensemble des activités sportives. L'entraide et l'assistance constituent l'un des principaux buts des associations ; c'est ici qu'il faut inscrire la préoccupation pour la santé.

\section{Les cotisations}

C'est autour de l'entraide et de l'assistance mutuelle que l'association organise toutes les activités relatives à son fonctionnement, notamment en ce qui concerne les prestations financières. Les cotisations des membres peuvent ressortir à la catégorie des "tontines", épargne personnelle tournante qui est collectée à chaque réunion, auprès de tous les membres et reversée à l'un d'entre eux, ou aux "caisses", qui correspondent aux cotisations remises à un trésorier et qui sont utilisées pour réaliser des objectifs fixés au départ : alimenter une caisse d'épargne qui peut distribuer des crédits, assister les membres en cas de maladie, de décès, organiser une fête, assurer le 
fonctionnement de la structure, etc. Ces "caisses" fondent les relations sociales, et les membres ne sont ensemble que parce qu'ils peuvent donner et attendre en contrepartie diverses formes de prestations.

14 Toutes les associations ne demandent pas de cotisations, c'est le cas notamment des associations religieuses et sportives qui peuvent se maintenir en dehors de prestations financières. Cependant, d'une manière générale, l'argent joue un rôle majeur dans la socialité associative. Il faut pourtant évaluer le montant des cotisations pour comprendre si l'aspect économique est dominant, ou s'il s'agit surtout de cimenter les relations sociales. On peut faire l'hypothèse que des sommes très élevées, compte tenu du niveau de revenu de ces populations, sont liées à un projet économique, tandis que des montants modiques indiqueraient plutôt une activité à caractère social. L'examen du tableau suivant nous permet de vérifier cette hypothèse.

Tableau 2 : Montant des cotisations déclarées (en FCFA)

\begin{tabular}{|l|l|l|l|l|l|l|}
\hline 0 & $<=5.000$ & $5000-15.000$ & $>15.000$ & Taux variable & Taux échelonné & Total \\
\hline 14 & 16 & 3 & 4 & 8 & 17 & 62 \\
\hline
\end{tabular}

15 Les cotisations sont à taux fixes, variables et échelonnés. Les taux variables permettent à chaque membre de verser un montant correspondant au niveau de ses revenus; ceux qui ont beaucoup d'argent versent des montants élevés, les autres, des sommes plus modestes. Mais chacun doit s'attendre en retour à des retombées proportionnelles au montant versé. Cette disposition illustre une des caractéristiques des associations: regrouper des personnes qui se sentent les plus proches en dépit des différences de revenus. Ainsi, par exemple, les membres d'une même ethnie restent apparemment solidaires en dépit des différences de revenus, et l'association est la manifestation de cette proximité.

16 S'agissant des associations à prestations échelonnées, elles mettent aussi ensemble des personnes de niveaux financiers différents. Cependant, des sous-groupes sont formés à l'intérieur de l'ensemble par rapport au niveau des revenus, les cotisations ne sont plus laissées à l'initiative des individus. Une même association peut regrouper deux ou trois catégories, dont l'une cotise à 10.000 CFA par personne, une autre à 5.000 , et une troisième à 2.000. Les retombées sont aussi proportionnelles aux versements des membres. Cependant, dans les deux cas, la participation aux rites, l'accès aux boissons, à la nourriture, et aux réjouissances se font selon le principe de la parité et non plus sur une base pyramidale, ce qui permet de maintenir l'illusion de l'égalité des membres. Ces systèmes permettent ainsi à chaque catégorie de membres d'épargner en fonction de sa catégorie sociale et de ses revenus personnels. Les sommes collectées sont généralement reversées aux membres à la fin de l'exercice. Les taux les plus élevés (25.000 et 50.000) existent dans certaines associations à des fins commerciales ou d'investissement.

17 A côté de ce que nous avons appelé cotisations et tontines, et qui est loin de recouvrir la totalité des activités financières des associations, il existe des "caisses" au sein des associations, sorte de mise en commun des ressources financières par les membres pendant une période bien déterminée. Cette mise en commun intervient généralement 
en cas de deuil ou de maladie. La nomenclature des "caisses" est variée, ainsi que le montre le tableau ci-dessous.

Tableau 3 : Les caisses et leurs montants déclarés (en CFA par personne et par an)

\begin{tabular}{|c|c|c|c|c|c|}
\hline \multirow[t]{2}{*}{ Caisses } & \multicolumn{3}{|c|}{ Taux fixes } & \multirow[t]{2}{*}{ Taux variables } & \multirow[t]{2}{*}{ Tota } \\
\hline & $<=5.000$ & $\begin{array}{l}5.000 \\
25.000\end{array}$ & $\begin{array}{l}25.000 \\
50.000\end{array}$ & & \\
\hline Secours/décès/assistance & 7 & 26 & 11 & 4 & 48 \\
\hline Transport & 6 & 3 & & & 9 \\
\hline Restauration & 1 & 1 & & & 2 \\
\hline Épargne & 1 & 1 & & 29 & 31 \\
\hline Investissement/affaires/ustensiles & 1 & & & & 1 \\
\hline Scolaire & & & & 13 & 13 \\
\hline Assurance vie & 6 & 1 & 1 & 1 & 9 \\
\hline Danse et fêtes annuelles & 1 & & & 1 & 2 \\
\hline Développement & 1 & 2 & & 4 & 7 \\
\hline Enseignement/évangélisation & & & & 1 & 1 \\
\hline Fonctionnement/adhésion & & 2 & & 1 & 3 \\
\hline Maladie/santé & 1 & 1 & & & 2 \\
\hline Main levée & & & & 1 & 1 \\
\hline Total & 18 & 11 & 12 & 54 & 95 \\
\hline
\end{tabular}

- La "caisse secours" sert à aider ceux qui organisent une fête, qui doivent prendre en charge les soins en cas de maladie, ou qui ont perdu un membre de leur famille nucléaire en ligne directe ascendante (père, mère), descendante (un enfant), ou un conjoint. A la fin de l'exercice budgétaire, s'il y a un excédent, il est redistribué aux membres au prorata des cotisations. Cependant, dans une des associations, les excédents sont gérés de la manière suivante : une partie des ressources est conservée comme fonds de caisse pour démarrer les activités de l'année suivante, l'autre partie est employée pour offrir à chaque membre un présent au cours de la fête de fin de l'année, appelée "fête de cassation".

- La caisse "Décès/assistance" : dans certaines associations, ces prestations sont du ressort de la caisse secours ; lorsqu'une caisse spécifique "décès-assistance" existe, c'est pour 
individualiser les prestations que l'on verse à celui qui est frappé par un deuil, ou par une maladie.

- La caisse "Transport" est destinée à financer les frais de transport de la délégation que l'association envoie pour assister le membre qui organise des obsèques, ou dont les obsèques ont lieu.

- La caisse "Restauration" permet de financer les repas et les boissons au cours des réunions ; en général, cette caisse est destinée à organiser le banquet de fin de cycle.

- La caisse "Epargne" permet de collecter l'épargne des membres de l'association pour une redistribution sous la forme de crédits à ceux qui ont besoin d'un emprunt. Les prêts sont remboursés moyennant un intérêt qui sert à rémunérer l'épargne. Tous les prêts doivent être remboursés au plus tard le jour de la "fête de cassation", et toute l'épargne, ainsi que les intérêts, doivent être reversés aux épargnants ce jour là. Un membre peut éventuellement emprunter auprès de cette caisse pour prendre en charge les soins d'un malade dont il a la charge.

- La caisse "Investissements/affaires/ustensiles" permet d'obtenir de l'argent destiné à un investissement quelconque : acheter de la vaisselle, des ustensiles de cuisines, constituer un fonds de commerce, etc ; une délégation est alors désignée par l'association pour s'assurer que l'argent est effectivement employé à ce à quoi il était destiné.

- La caisse "scolaire" fonctionne sur le même mode que la précédente, et est spécifiquement destinée à prendre en charge les frais scolaires des enfants des membres de l'association.

- L'assurance vie a été instaurée au sein de certaines associations pour pallier les insuffisances de la caisse secours, devant la modicité des sommes perçues par ceux qui reçoivent les prestations liées à un décès. Elle est sollicitée en cas de deuil, lorsque meurt un membre de l'association; l'argent est versé à sa famille.

- La caisse "Danses et fêtes annuelles" est utilisée pour la "fête de la cassation" qui signe une fin de cycle, mais aussi, pour des associations à caractère ethnique, pour des manifestations collectives du groupe : une des associations étudiée utilise cette caisse pour la fête de fin de cycle, précédée par un congrès, fête au cours de laquelle les conjoints sont invités, et pour organiser des excursions.

- La caisse "Développement", dans les associations d'originaires, sert à financer des projets de développement dans les terroirs d'origine ; parfois des amicales d'anciens élèves l'utilisent aussi pour venir en aide à leur ancien établissement scolaire.

- La caisse "Enseignement/évangélisation" se retrouve dans des associations religieuses comme l'Association catholique des femmes et set à organiser des séances d'évangélisation, à acheter des documents, etc.

- La caisse "Fonctionnement/adhésion" sert aux membres du bureau de l'association pour financer l'achat du matériel de bureau, faire des photocopies, etc ; sont également versées dans cette caisse les pénalités, les amendes infligées aux retardataires, aux absents, aux chahuteurs, à ceux qui ne portent pas l'uniforme, ou à ceux qui ont été surpris en train de le porter hors des manifestations de l'association.

- La caisse "Maladie/santé" se retrouve dans quelques associations qui ont individualisé les interventions relatives à la santé. Cet argent, provient souvant de la "caisse secours" et est alors destiné uniquement à la prise en charge des soins des membres malades, ou des membres de leur famille selon une nomenclature précise.

- La "Main levée" est une quête spontanée ; en cas de maladie, elle vient suppléer la modicité des interventions de la "caisse secours", ou de la "caisse maladie". Quand on en sent la nécessité, et qu'elle est proclamée, elle devient obligatoire pour tous les membres de l'association. 
Le tableau ci-dessous résume les différents types de caisse et de prestations.

Tableau 4 : Les "caisses" des associations

\begin{tabular}{|c|c|}
\hline Types de caisse & Utilisations \\
\hline $\begin{array}{l}\text { Secours solidarité/ } \\
\text { Aide/décès/ } \\
\text { Mutuelle }\end{array}$ & $\begin{array}{l}\text { Sert pour le fonctionnement, pour secourir les membres en cas de deuils, } \\
\text { évacuer les membres décédés, aider financièrement les conjoints, les } \\
\text { enfants légitimes des membres et les parents en cas de décès }\end{array}$ \\
\hline Santé ou maladie & Secours en cas de maladie \\
\hline Enseignement & Evangélisation \\
\hline Transport & $\begin{array}{l}\text { Sert au transport des membres qui accompagnent la famille en cas des } \\
\text { deuils }\end{array}$ \\
\hline Épargne & $\begin{array}{l}\text { Assurer les fêtes annuelles dans les associations, accorder des emprunts } \\
\text { aux membres en cas de besoins }\end{array}$ \\
\hline Restauration et fête & Organiser les banquets à la fin de l'année \\
\hline Scolaire & Epargne pour la préparation de la rentrée scolaire \\
\hline Vaisselle & Permet aux membres d'acquérir des ustensiles de cuisine et de la vaisselle \\
\hline Danse & Achat d'instruments de musique, d'accessoires de danse \\
\hline $\begin{array}{l}\text { Adhésion et fonds } \\
\text { de roulement }\end{array}$ & Fonctionnement du bureau de l'association \\
\hline Développement & Promotion et développement des villages \\
\hline $\begin{array}{l}\text { Investissement et } \\
\text { affaires }\end{array}$ & $\begin{array}{l}\text { Création des petites activités, par exemple, assainissement et } \\
\text { électrification du quartier }\end{array}$ \\
\hline Assurance vie & Assurer les membres en cas de décès \\
\hline Main levée & Appui à la caisse secours \\
\hline
\end{tabular}

La prise en charge de la santé

19 Onze des soixante huit personnes interrogées sont membres d'associations dont les cotisations varient entre 25.000 et 50.000 FCFA par personne et par an et dont la caisse secours est aussi destinée à la prise en charge des soins de santé ; l'intervention de l'association peut être alors substantielle en cas de maladie. Mais la "caisse secours" est destinée à d'autres interventions, et aucune caisse spécialisée ne reçoit des versements d'un montant aussi important. Une seule caisse affiche un taux de cotisation individuel de 12.000 FCFA par an, soit un versement de 1.000 francs par mois et par membre. Il 
apparaît donc nettement que, d'une manière générale, la santé ne fait pas l'objet de prestations très importantes.

Seules cinq personnes mentionnent une caisse spécifiquement dédiée aux soins médicaux. La majorité des personnes interrogées et ayant répondu, soit 41, évoquent le recours à la "caisse secours" pour faire face aux dépenses de santé. Cependant la "caisse secours" étant destinée aussi à d'autres fins, elle ne peut assurer une couverture aussi substantielle qu'une caisse spécialisée. Les personnes dans le besoin, y compris donc en cas de maladie, peuvent cependant bénéficier d'un crédit auprès de l'association, ce qui permet de disposer rapidement de liquidités, tout en créant une certaine précarité. Le recours à un crédit auprès de l'association entraîne le sociétaire dans un cycle d'endettement dans la mesure où les taux d'intérêt peuvent être très élevés, 5 à $10 \%$ par mois, soit de 60 à $120 \%$ par an. L'impression demeure donc, d'une implication marginale des associations dans la prise en charge des soins de santé.

L'assistance aux membres est l'une des activités principales des associations même si certains aspects, tels que la promotion et l'aménagement des zones rurales, l'évangélisation et la création de réseaux d'amitié entre anciens camarades de collège ou d'université apparaissent aussi. Quant aux activités secondaires, elles sont souvent orientées vers des manifestations culturelles, les rencontres annuelles lors des congrès ou la "fête de cassation" qui sanctionne la fin des activités annuelles de l'association et le démarrage d'un nouvel exercice. L'assistance en cas de maladie est modique, et se doit d'être complétée par d'autres sources financières.

La plupart des associations pratiquent l'assistance sous forme financière et de visites collectives ou individuelles. Elles organisent parfois des séances de prières. Ce qui n'exclut pas par ailleurs qu'il y a des associations dont l'essentiel de l'assistance se limite strictement à des visites en petits groupes en fonction des affinités. En fait, on peut relever une contradiction entre l'intervention affichée des associations pour les soins de santé, presque toutes l'intégrant dans leur démarche, et la modicité des sommes remises au malade. Une question surgit donc : $\mathrm{y}$ a-t-il véritablement assistance des associations en cas de maladie? Les sommes remises au malades s'échelonnent le plus souvent entre 10.000 et 20.000 FCFA. Elles ne permettent donc pas une prise en charge substantielle des frais médicaux ou d'hospitalisation. Les associations n'interviennent pas non plus en situation d'urgence, car toutes les maladies ne sont pas prises en charge ; les sociétaires doivent en effet convenir qu'un cas est sérieux pour intervenir. Cela suppose donc qu'ils se sont réunis auparavant, ou qu'une démarche a été entreprise auprès des membres du bureau de l'association pour solliciter une aide. L'assistance privilégie les hospitalisations et, dans une moindre mesure, les autres formes de soins souhaitées par les membres. Les associations ethniques, les amicales, régionales, sportives, socio-professionnelles privilégient plus que les autres les soins médicaux à l'hôpital. Les associations de famille, religieuses, de quartier et certains regroupements ethniques laissent leurs membres faire appel aux recours thérapeutiques de leur choix. On peut parler d'une tendance à privilégier l'hôpital, et à ne prendre en charge que les maladies identifiées à l'hôpital

Les aides versées apparaissent surtout sous la forme de forfaits quelle que soit la gravité de la maladie. Une minorité d'informateurs signale une évaluation de la gravité de la maladie par les membres de l'association qui permettrait de fixer les montants à verser. Certaines associations choisissent de faire varier les taux, en fonction de l'état du malade, mais aussi du recours thérapeutique choisi et de l'efficacité accordée à ce 
recours. Il s'agit-là d'une tendance minoritaire, qui montre cependant que cette préoccupation n'est pas toujours absente. Il faut quand même rappeler que cette assistance ne constitue pas l'unique forme d'intervention des associations dans le domaine de la santé, les emprunts auprès de la "caisse d'épargne" sont toujours possibles, de même qu'un recours à la "main levée".

Les procédures d'octroi sont connues : remettre à un membre de la famille, faire faire un constat par un membre de l'association ou recevoir le témoignage de la famille, vérifier le dossier médical. La vérification de papiers tels que les ordonnances, les billets d'entrée et de sortie sont les principaux moyens utilisés par les associations. Ces procédures prouvent que l'association ne peut intervenir en urgence, puisque l'ensemble des procédures de contrôle nécessaires au déblocage des fonds prend du temps. Cependant, en règle générale, le peu d'importance accordée à la vérification des papiers prouve que les sociétaires ont confiance dans le jugement et la bonne foi des membres de leur organisation. Les procédures d'octroi, même simplifiées, peuvent prendre du temps; et la modicité des sommes accordées ne justifie pas une procédure très complexe. La plupart des personnes rencontrées affirment que les membres de l'association n'exigent pas de justificatifs de la transaction financière en cas de maladie. Seuls 7 informateurs ont confirmé que leur association exigeait des justificatifs. En règle générale les associations se contentent d'une déclaration orale, l'argent étant remis par une délégation de l'association, ou à un membre de la famille du malade au cours d'une réunion.

Certaines amicales et associations ethniques remettent cette aide financière au malade lui-même, à un autre membre de l'association ou tout simplement, forment une délégation de quelques membres qui rend visite au malade et lui remet alors l'argent séance tenante. C'est le cas des associations de quartier par exemple. La préoccupation financière est somme toute relative au sein des associations où la comptabilité est contraignante. Si l'argent n'est pas la principale forme de soutien de l'association à ses membres en cas de maladie, et si les prestations financières ont un caractère surtout social, la mobilisation autour de la maladie se manifeste surtout par des visites au malade, des séances de prière, et d'autres manifestations religieuses telles que les chants, les messes et les exorcismes.

Les visites s'effectuent en groupe, par délégation de quelques membres, ou de façon individuelle. Elles s'accompagnent souvent des conseils, notamment sur les choix thérapeutiques, des dons en nature, ou en espèces, par exemple en cas de cotisation exceptionnelle de "main levée". Un seul informateur mentionne le cas d'une association qui n'envoie pas de délégation auprès des malades. Toutes les associations sont donc préoccupées par la santé de leurs membres et réagissent en conséquence. On retrouve ainsi le contraste entre une préoccupation constante à propos de la maladie mais qui ne se traduit que par des aides financières très modestes. On ne saurait expliquer cet état de fait par la pauvreté, des sommes beaucoup plus substantielles étant allouées pour les cérémonies funéraires, pour l'organisation de fêtes en général, ou pour la fête de fin de cycle. Il est aussi étonnant qu'une population de fonctionnaires, de cadres et de commerçants, comme celle rencontrée à Biyem Assi, n'ait pas envisagé des formes d'assurance sociale, hormis une seule association qui était en train d'y réfléchir lors de notre enquête. Des obsèques fastueuses, un banquet de fin de cycle sont des priorités, au vu des sommes investies dans l'organisation de ces manifestations. 


\section{AUTEURS}

\section{SÉVERIN CÉCILE ABÉGA}

Institut de recherche en socio-anthropologie, Université Catholique d'Afrique Centrale, BP 11628 Yaoundé - Cameroun icy.ucac.fssg-fthnk@camnet.cm

\section{NOËL SOLANGE NGO YEBGA}

Institut de recherche en socio-anthropologie, Université Catholique d'Afrique Centrale, BP 11628 Yaoundé - Cameroun yebs20@yahoo.com 